\title{
A Cross-Layer Optimization Framework for Energy Efficiency in Wireless Sensor Networks
}

\author{
Karuna Babber, Rajneesh Randhawa \\ Computer Science Department, Punjabi University, Patiala, India \\ Email: ka runababber.kb@gmail.com
}

How to cite this paper: Babber, K. and Randhawa, R. (2017) A Cross-Layer Optimization Framework for Energy Efficiency in Wireless Sensor Networks. Wireless Sensor Network, 9, 189-203.

https://doi.org/10.4236/wsn.2017.96011

Received: June 2, 2017

Accepted: June 25, 2017

Published: June 28, 2017

Copyright $\odot 2017$ by authors and Scientific Research Publishing Inc. This work is licensed under the Creative Commons Attribution International License (CC BY 4.0).

http://creativecommons.org/licenses/by/4.0/ (c) (i) Open Access

\begin{abstract}
We consider the extension of network lifetime of battery driven wireless sensor networks by splitting the sensing area into uniform clusters and implementing heterogeneous modulation schemes at different members of the clusters. A cross-layer optimization has been proposed to reduce total energy expenditure of the network; at network layer, routing is done through uniform clusters; at MAC layer, each sensor node of the cluster is assigned fixed or variable time slots and at physical layer different member of the clusters is assigned different modulation techniques. MATLAB simulation proved substantial network lifetime gains.
\end{abstract}

\section{Keywords}

Clustering, Cluster Heads, Border Nodes, Base Station, Cross-Layer Design, Physical Layer, MAC, Routing Layer, Packet Size, Modulation, Quality of Services (QoS), Wireless Sensor Networks

\section{Introduction}

In recent years wireless sensor networks (WSNs) are gaining momentum in almost every facet of life whether it is health care, home security, forest area supervision, monitoring earth movement or battle field surveillance. In particular, the core objective of WSN applications is to reliably detect the event and send the collective information to the base station or sink. But the main challenge to achieve this objective is posed by limited energy of sensor nodes which are generally battery driven. Till date a significant amount of research on energy efficient protocols has been come up but to the best of our belief none of the protocol is best suited for WSNs.

Wired networks follow traditional 7-layered Open System Interconnection (OSI) reference model with each layer responsible for explicit task like Physical 
layer is responsible for the transmission of raw bits, modulation schemes, data rate and transmission power of the nodes which effect the overall power consumption of the network. At data link layer again different sources of energy wastage like overhearing, idle listening, collision and transmission of control packets overhead are there. At network layer best-shortest route and routing functionalities consumes much of the energy of the network. With this way all the seven layers with different functionalities wastes considerable amount of network energy. In wired networks because of unlimited power supply, working with these protocols does not pose problems but for battery driven networks like WSNs such issues require serious re-consideration of already existing layered architecture and as such a cross-layer approach may prove prolific for WSNs.

\section{Prior Work}

The Cross-layer design may be defined as, "The breaking of OSI hierarchical layers in communication networks [1]. The intent of cross-layer design is to simply utilize information of different layers of OSI/TCP models and jointly optimize the performance of these layers for improving QoS of wireless networks. In two different ways we can opt for cross-layer approach i.e. static cross-layer approach in which we can utilize known characteristics of layers and jointly optimize their performances or co-calibrate these layers by creating new interfaces or interactions among the layers. Secondly by implementing dynamic cross-layer designs [2] which can respond and adapt to network conditions by exposing internal information of layers to built new interfaces. The great challenge behind implementing cross-layer design lies in the fact that sometimes the creation of new interfaces may lead to unexpected dependencies which otherwise can't be predicted at simulation or testing level. Secondly in communication systems, many random variations may occur at top (application layer) and bottom (physical) layers. As WSNs are supposed to have extremely lengthy network lifetime and make efficient utilization of their limited perished power sources (generally battery), much of the recent research is on adopting cross-layer approach. A brief overview of recent work on cross-layer optimization for minimizing energy consumption is given below:

1) In [3] authors suggested unified cross-layer module (XLM) in which common layered functionalities are combined together to achieve energy efficient communication in wireless sensor networks. Though XLM performs receiver based contention but every node here is to decide about the participation in the communication which itself increases the broadcasting burden of RTS and CTS packets. As a result of which energy cannot be saved to a desirable length.

2) A cross-layer solution based on duty cycling approach along with new kind of active wake-up circuit used in the communication [4]. In the network set-up phase, a power detector is used which helps in awakening the nodes whenever the transmission of a neighbor is scheduled. This reduces idle listening period but raises complexity of circuitry design of sensor nodes. 
3) A two-level node activity scheduling scheme for energy efficient communication has been proposed by [5]. Coarse-grained and Fine-grained scheduling has been implemented on the working nodes of each sub grid and all other nodes are turned to sleep mode. Though the schemes are good enough for static and scenario aware networks but what if the network changes dynamically. It is difficult to stay awake the other nodes, if there is a message to transmit or receive.

4) A cross-layer solution for real time data delivery has been proposed by [6]. The average packet service time along with a weighted cost function is used to choose a most appropriate routing path for sensor nodes to transmit. This further helps to reduce end-to-end delay and thereby enhances energy efficiency. But to maintain strict end to end delay deadlines is not viable in all the cases.

5) An adaptive modulation coding scheme (AMC) has been proposed in [7] wherein AMC provides " $k$ " packets of finite length to form a queue at data link layer. The authors used values of $k, \mathrm{~m}$ (Nakagami parameter) and received gamma value to achieve targeted packet error rate (PER). The Markov chain model along with Nakagami parameter is further used to adjust channel fading and estimating channel state transition probabilities.

6) A cross-layer approach with an adaptive modulation \& coding (AMC) at physical layer and truncated automatic repeat request (ARQ) at data link layer has been proposed in [8]. The packet length and average round trip time (RTT) at data link layer together decides the delay constraints whereas after retransmissions, the probability of packet loss was calculated which then considered as the PER upper bound. To optimize the system performance in terms of transmitted bits per symbol, received signal to noise ratio (SNR) and PER upper bound has been considered.

7) In [9] authors suggested energy efficient modulation schemes for coded and un-coded systems. Authors further suggested that for a known number of bits to be transmitted, an optimal constellation size can be calculated which further helps to reduce energy consumption by reducing total transmission. It is clearly shown that rated adaptation can lead to significant energy saving of the network.

8) An optimized data transmission method is proposed in [10] for Time Division Multiple Access (TDMA) network with the assumption that no interference takes place. It is shown in that by implementing new coding schemes, decoder energy can also be reduced significantly. Authors further added that by appropriately considering modulation/coding scheme and receiver decoder complexity, network lifetime can be extended.

9) In [11] authors propose duty cycling within the network by turning off the transceivers when they are not needed i.e. during back-off periods. Authors further claimed power saving of the network by reducing idle listening and decreasing number of collisions during transmissions.

10) An energy aware cross-layer data gathering, RMC protocol for wireless sensor networks is presented in [12] wherein to reduce the overheads integration 
of routing, MAC and clustering of nodes is done. Cross-layer integration of MAC and routing layers conserve significant amount of energy of WSNs as is suggested in [13] [14], but the work provides only analytical results without any communication design.

11) Receiver based routing has been proposed in [15] wherein the selection of next hop is based on cross-layer modularity between MAC and routing layers. The issues of latency and multi hop performance during cross layer information exchange are also discussed.

12) A Cross-Layer Power Control Algorithm (CLPCA) and a Power Control Based Real-time Routing Protocol (PCBRRP) are suggested by [16]. In this protocol authors dynamically adjusted the transmission power of nodes and the selection of next hop node is based on its residual energy and link quality.

13) Issues of channel allocation, routing and data rate control in layered architecture have been addressed in [17]. The varying channel link capacity due to interference put an impact on topology change and if the channel is multicast, due to fading it becomes even more challenging to use exiting protocols. To overcome such problems authors suggested cross-layer optimization between transport, MAC and physical layers.

14) In [18] authors suggested cross-layer interaction by combining two communication protocols: data aware reporting tree construction and node aware scheduling at network and MAC layers. Authors used single-hop cluster based topology to simulate the throughput performance, though it can also be used on different topologies.

\section{Proposed Uniform Clustering with Low Energy Adaptive Hierarchy (UCLEAH) Model}

The entire sensing area is divided into ordinary sensor nodes, cluster heads and border nodes. The ordinary sensor nodes monitor the environment and transmit its data to their respective cluster head nodes. The cluster head nodes in turn gather data from all ordinary sensor nodes of the cluster compress and aggregate the received data and then forward it to the border nodes. The border nodes in turn transmit the sensed data to Base station/sink. These border nodes are selected in such a way that these are supposed to be closer to Base station.

a) Routing information: The sensing area can be partitioned into any defined angle say at every $30,45,90$ or 120 degrees and then the average distance of sensor nodes is taken into consideration to further divide them into uniform clusters. The main focus of this algorithm is to reduce hop distance among sensors and to reduce energy consumption during data transmission and reception.

b) Route set-up phase: The objective of this phase is to create uniform clusters, selecting cluster heads and border nodes. During the set-up phase, the base station collects the information of the position of all sensor nodes within the sensing area along with their unique identification number. To form uniform 
clusters and choosing cluster heads and border nodes, following steps are to be followed:

1) Initial energy of all sensor nodes is same say $E_{\text {init }}=2$ (say).

2) The Base station is assumed to be located at a fixed position say $(X, Y) \mathrm{m}$ and have unlimited communication power with unlimited energy access.

3) Each node using its Global Positioning System (GPS) knows its location within the sensing area and sends its location along with its unique Identification Number (ID) to the Base station.

4) To calculate the "Centre Location" $(C)$ with co-ordinates $C_{x} \& C_{y}$ of the sensing area, equation $\frac{\sum_{i=1}^{n} X_{i}}{N}$ where $X_{i}$ is the co-ordinate of sensor node $i$ can be used.

5) To calculate the accurate distance (d) of each sensor node from the Centre location (C), equation $d=\sqrt{\left(C_{x}-x\right)^{2}+\left(C_{y}-y\right)^{2}}$ can be used, where $(x, y)$ are the location co-ordinates of a sensor node.

6) To form uniform clusters, sensing area can be split into any defined angle and accordingly different equations can be formed like:

Cluster $1^{\text {st }}$, for different values of $x, y$ lies between: $C_{y} \leq y \leq C_{y}+\tan \theta_{1}\left(x-C_{x}\right)$.

Cluster $2^{\text {nd }}$, for different values of $x, y$ lies between: $C_{y}+\tan \left(\theta_{2}-\theta_{1}\right)\left(x-C_{x}\right) \leq$ $y \leq C_{y}+\tan \left(\theta_{2}-\theta_{1}\right)\left(x-C_{x}\right)$.

Cluster $3^{\text {rd }}$, for different values of $y, x$ lies between: $C_{x} \leq x \leq C_{x}+\tan \left(\theta_{3}-\theta_{2}\right)(y$ $-C_{y}$ ) and so on $\cdots$

For Cluster $\mathrm{n}$, for different values of $x$ and $y$ lies between: $C_{y}+\tan \left(\theta_{n}-\theta_{n-1}\right)(x$ $\left.-C_{x}\right) \leq y \leq C_{y}$.

7) After cluster formation, for selection of cluster heads, mean distance of cluster sensor nodes from the Centre location $(C)$ can be calculated as follows:

$$
\begin{aligned}
& C_{H 1}=\frac{d_{11}+d_{12}+\cdots+d_{1 p_{1}}}{p_{1}} \\
& C_{H 2}=\frac{d_{21}+d_{22}+\cdots+d_{2 p_{2}}}{p_{2}} \\
& \cdots \\
& C_{H n}=\frac{d_{n 1}+d_{n 2}+\cdots+d_{n p_{n}}}{p_{n}}
\end{aligned}
$$

where $p_{1}, p_{2}, \cdots, p_{n}$ are the number of sensor nodes within the cluster.

8) Node whose distance from Centre Location $(C)$ is highest among all within the cluster can be chosen as a Border node, as it ensures that this particular node (Border node) is farthest from Centre Location $(C)$ but will be near to the Base station/sink.

Figure 1 depicts cluster architecture of the sensing area. The Base station broadcasts the routing information and time schedule in the form of fixed or variable TDMA slots to all the members of the clusters i.e. ordinary sensor nodes, cluster heads and Border nodes. Hence each sensor node has its own routing table and TDMA schedule, to follow. 


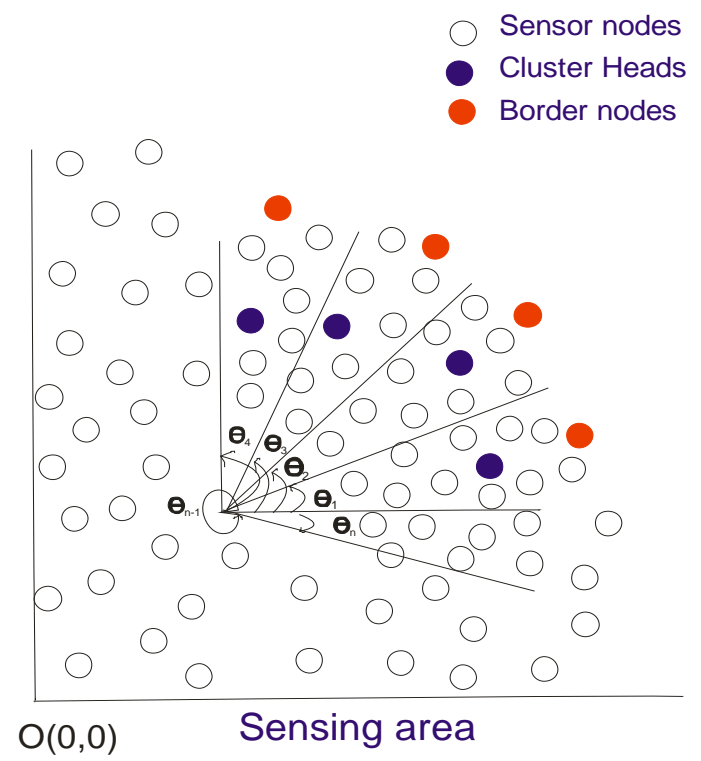

Figure 1. Cluster architecture.

\section{Channel and Physical Layer Model}

The proposed UCLEAH algorithm provides a centralized network topology and as the circuitry design of sensor nodes is simple with limited battery and processing capabilities, the channelization can be done by the base station only.

\section{Proposed Physical Layer Frame Structure}

The 802.11 Physical layer frame structure consists of 4 bytes preamble, 1 byte PHY Header, 1 byte delimiter and up to 127 bytes of payload data. The preamble is composed of 10 repeats of "short training sequence" and 2 repeats of "long training sequence" generally for channel estimation as well as frequency and time synchronization with the receivers whereas the Header gives packet configuration like its format, length and data rates. The payload data contains actual data to be transmitted. It also contains different types of frames like management, data and control frames. Each frame further consists of MAC header, payload information and frame check sequence.

Optimizing data packet length in wireless networks to minimize energy consumption has found considerable attention in literature [19] [20]. In [21] estimated bit error rate is used to optimize the packet size whereas in [22] error correction bits added on packet size improves energy efficiency. In this paper we try to optimize the packet length by removing preamble bytes as we don't need symbol synchronization due to pre-determined hop distance among sensor nodes and cluster heads (using proposed UCLEAH algorithm). Secondly the need of management, data and control frames also doesn't arise here because of the pre-determined route and receiver (cluster head) address. Instead of preamble, a pattern sequence ( 11 or 00$)$ for channel estimation can be added for better synchronization between transmitting sensor node and cluster head. Figure 2 shows the proposed packet structure. 


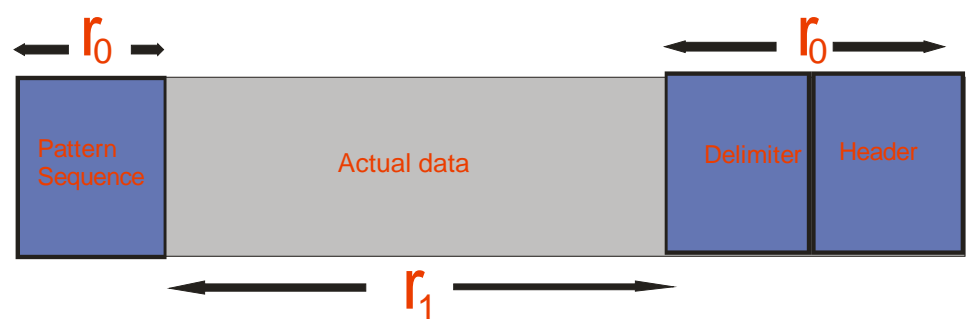

Figure 2. Packet structure.

1) Energy model: The total energy consumption model for transmitting single data packet is given in [23] as:

$$
E=\alpha E_{r x} d^{n}+E_{\text {fixed }}
$$

where $\alpha$ is the amplifier co-efficient, $E_{r x}$ is the energy consumption during the reception of per data packet, $d$ is hop-distance and $E_{\text {fixed }}$ is the fixed energy required by transmitter/receiver to transmit or receive one data packet. Time taken to transmit a data packet of $r$ bits is given by:

$$
T_{r}=\frac{r_{1}}{b B}+\frac{r_{0}}{B}
$$

where $r_{1}$ is for actual data bits and $r_{0}$ are overhead (delimiter/header and pattern sequence) bits and $B$ is the signal bandwidth. Total energy consumed during transmission/reception per data packet in terms of bits can be given as:

$$
\begin{gathered}
E=\left(E_{t x}+E_{r x}\right) T_{r} \\
E=\left(E_{t x}+E_{r x}\right)\left(\frac{r_{1}}{b B}+\frac{r_{0}}{B}\right)
\end{gathered}
$$

The above equations clearly depicts that the total energy consumption during transmission and reception of a data packet is directly proportional to the number of bits contained in data packet and the hop distance. Thereby the proposed packet structure and UCLEAH algorithm both helps to achieve lesser total energy consumption of the network.

2) Allotment of TDMA slots and capacity enhancement through frequency re-use: Using UCLEAH, sensor nodes which lie in the diameter of 30 or 35 meters from cluster heads may be provided fixed TDMA slots but sensor nodes which are little far i.e. above 35 meters of diameter from their respective cluster heads may be provided variable TDMA slots. Secondly capacity enhancement through frequency re-use of the sensing network can be done using the following equation:

$$
k * u
$$

where $k$ is the enhancement due to multiplexing using TDMA slots in a cluster and $\mathrm{u}$ is the number of adjacent clusters in the sensing area. The base station or sink allots time slots and assigns frequency channels using above equations to all the sensors of the network.

3) Proposed modulation schemes: As is evident from Equations (1) to (4) that energy consumption is directly proportional to hop distance. The sensor 
nodes might be at different distances from their respective cluster heads, so employing single modulation scheme on all members of the sensing network surely won't be a good idea. Using energy consumption and symbol error formulas given by [24] [25] in the Table 1 below, two scenarios have been drawn:

\section{Scenario I:}

- Each modulation scheme (Homogenous modulation) applied on all the members of the cluster.

- Constant Bit Error Rate (BER) along with the ratio of energy per bit to the noise variance $\left(E_{b} / N_{o}\right)$ for different modulation schemes maintained.

- Each modulation scheme applied in White Gaussian Noise Channel (AWGN) environment.

In this scenario, when BPSK, 8-PSK modulation techniques (being very simple to generate and demodulate) are applied on all the members of the cluster, these performed good in terms of energy efficiency but proved fairly in-effective in terms of channel throughput and data rates whereas when 16PSK and 4QAM modulation techniques (being complex to design and demodulate) are applied on all cluster members, these techniques provided high channel throughput but consumed good chunk of energy of the network.

\section{Scenario II:}

- Different modulation schemes (Heterogeneous modulation) applied on different members of the cluster.

- Constant Bit Error Rate (BER) along with the ratio of energy per bit to the noise variance $\left(E_{b} / N_{o}\right)$ for different modulation schemes maintained.

- Each modulation scheme applied in White Gaussian Noise Channel (AWGN) environment.

In this scenario, it is observed that when the information transmitted with in a distance of up to 35 - 40 meters all the modulation schemes performed almost in a similar manner whereas when the distance increases say above 40 meters BPSK, 8PSK performed poorly in terms of channel throughput on the other hand 16PSK or 4QAM modulation schemes provided high channel throughput but are little expensive in terms of consumption of energy.

Table 1. Table of energy consumption and symbol error formulas.

\begin{tabular}{|c|c|}
\hline Modulation Scheme & $\begin{array}{l}\text { Energy consumption along with symbol error } \\
\text { estimation formulas }\end{array}$ \\
\hline BPSK & $Q\left(\sqrt{\frac{2 E_{s, R X}}{N_{0}}}\right)$ \\
\hline QPSK & $2 Q\left(\sqrt{\frac{2 E_{S, R X}}{N_{0}}}\right)\left((1-0.5 Q) \sqrt{\frac{E_{S, R X}}{N_{0}}}\right)$ \\
\hline M-PSK & $2 Q\left(\sqrt{\frac{4 E_{S, R X}}{N_{0}}} \sin \left(\frac{\pi}{M}\right)\right)$ \\
\hline M-QAM & $1-\left(1-2\left(1-\frac{1}{\sqrt{M}}\right) Q\left(\sqrt{\frac{3}{(M-1)} \frac{E_{S, R X}}{N_{0}}}\right)\right)^{2}$ \\
\hline
\end{tabular}


The focus of this paper is to extend the network lifetime to maximum and also to balance the entire network in terms of channel throughput and data rates. It is therefore, recommended that sensor nodes within the diameter of 35 to 40 meters be run on BPSK or 8PSK modulation schemes and when the distance between sensor nodes and their respective cluster head and cluster head and border node is above 40 meters 16PSK or 4QAM modulation techniques should be implemented. With this way we cannot only successfully sense the area for much greater time but also with effective data rates and channel efficiencies.

\section{Performance Analysis}

In this section, the base station controlled uniform clustering of the sensing area is done in accordance with the proposed UCLEAH algorithm and clusters have been formed at $\theta=30^{\circ}$ and $45^{\circ}$ respectively. Figure 3 and Figure 4 shows formation of clusters with cluster heads and border nodes of each cluster.

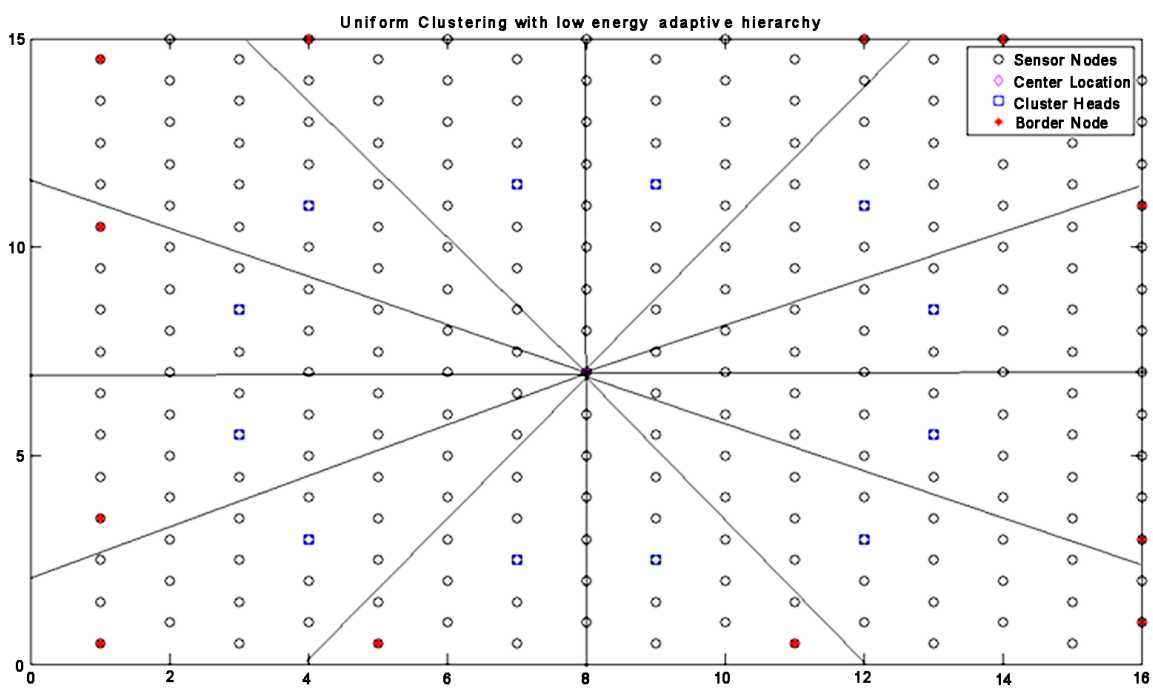

Figure 3. Uniform clustering at $\theta=30^{\circ}$ using UCLEAH algorithm.

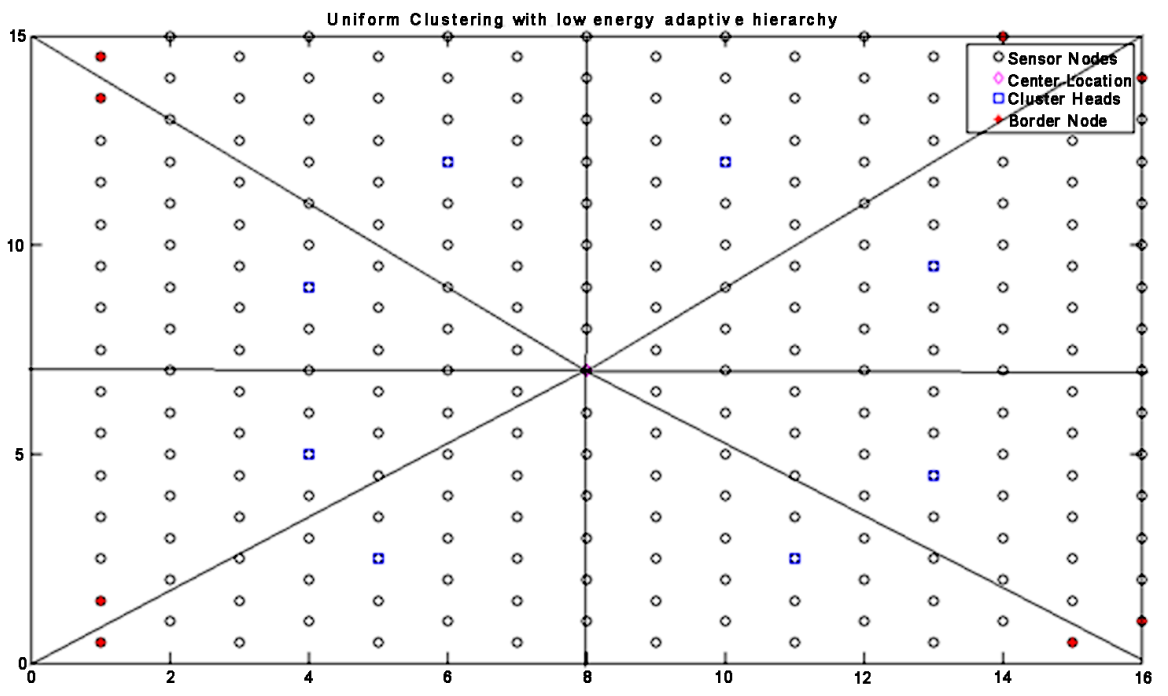

Figure 4. Uniform clustering at $\theta=45^{\circ}$ using UCLEAH algorithm. 
All the optimizations are based on numerical equations and are performed in MATLAB. Default parameters used during simulation are given as Table 2 under:

In simulation, for a fixed distance and constant bit error rate (BER) of $10^{-5} \mathrm{a}$ range of noise values from $N_{o}$ to $128 N_{o}$ has been chosen for the above mentioned modulation schemes. Figure 5 shows the energy consumption Vs Noise of different modulation schemes at a fixed distance of $30 \mathrm{~m}$.

\section{Effect of Packet Size}

It is observed that for any modulation scheme, the probability of receiving a data packet decreases with the increase in packet size. Therefore, small packet sizes may help to increase energy efficiency. However it is true only if no packet overhead is considered. Figure 6 shows the trade-off between the energy consumption and varied packet sizes (with different packet overheads).

The trade off in packet size with or without overhead can be seen in the above figure. When packet overhead is zero or minimum, energy tends to decrease but with the rise in packet overheads, the packet size also increases.

Table 2. Parameters used in the models.

\begin{tabular}{cc}
\hline Description & Value \\
\hline Radio cost $\left(E_{\text {fixed }}\right)$ & 2.80 mcJ per symbol \\
Packet size $(r)$ & 350 bits \\
Overhead bits $\left(r_{0}\right)$ & $60 \mathrm{bits}$ \\
Carrier frequency $(f)$ & $2.4 \mathrm{GHz}$ \\
Amplifier co-efficient $(\alpha)$ & 0.02 \\
Signal bandwidth $(B)$ & $100 \mathrm{kHz}$
\end{tabular}

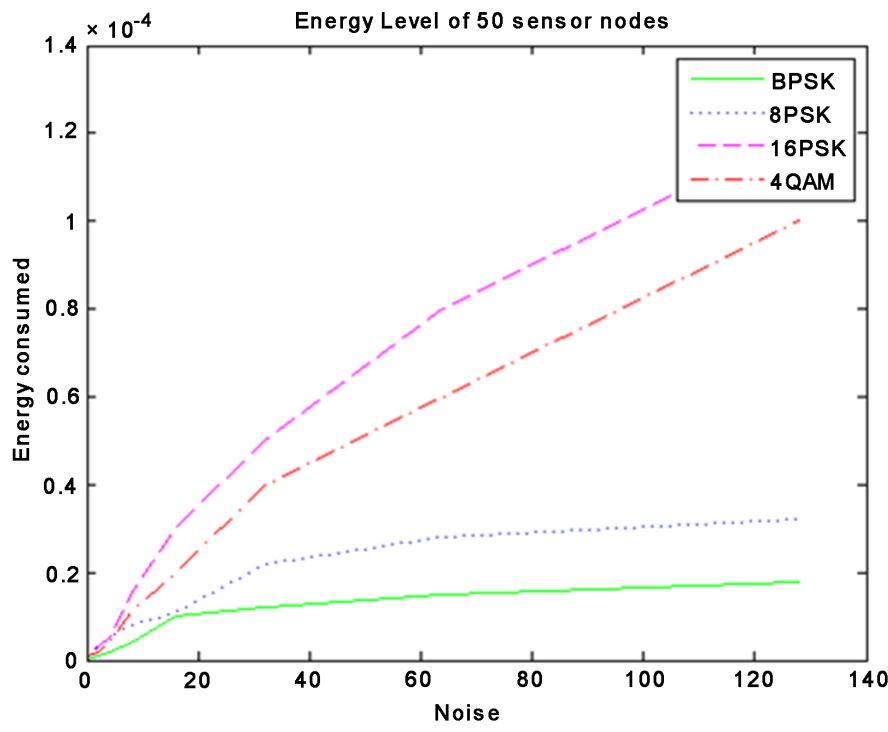

Figure 5. Energy consumption Vs Noise at a fixed distance of 30 meters. 


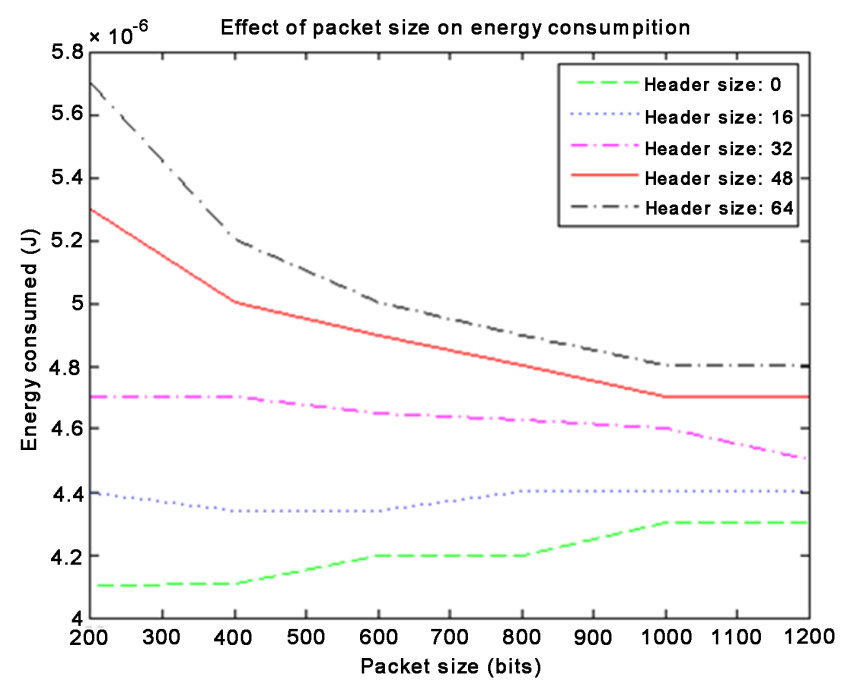

Figure 6. Energy consumption during varied packet sizes (different packet overheads).

\section{Optimal Modulation Schemes}

Equations (1) to (4) clearly shows that distance and transmission time are directly proportional to the energy consumed. Figure 7 gives us the fair idea about energy consumption at varied transmission time for short distance.

By implementing above mentioned two scenarios through simulation (see Figure $8 \&$ Figure 9), it has been found that when the transmission distance is less i.e. about $40 \mathrm{~m}$, energy consumed by BPSK, 8 PSK, $16 \mathrm{PSK}$ and $4 \mathrm{QAM}$ is almost same but when the distance increases say above $40 \mathrm{~m} \mathrm{16PSK}$ and 4QAM modulation schemes consumes more energy as compared to BPSK or 8PSK. Although BPSK or 8PSK modulation schemes are simple to design and demodulate but channel utilization and bandwidth efficiency of both these techniques is far less than 16PSK or 4QAM. On the other hand complex circuitry design of 16PSK or 4QAM techniques needs more processing capabilities of sensor nodes which in many cases may not be possible and feasible too.

Due to this trade off, it is highly recommended through this paper that to balance the entire network, a heterogeneous modulation technique may be implemented on the sensing area i.e. sensor nodes which falls into a diameter of up to 40 meters from their respective cluster heads may transmit data using BPSK or 8PSK modulation techniques and all other transmission which is above 40 meters (i.e. from cluster heads to border nodes and border nodes to base station) may use 16PSK and 4QAM modulation schemes. This way not only network lifetime can be extended but higher data rates can also be achieved.

To achieve desired results optimization of above mentioned parameters can be done according to the applicability and resources of WSNs.

\section{Conclusions}

In this paper we explored physical, MAC and routing layer parameters to enhance the network lifetime of wireless sensor networks. Through simulation 


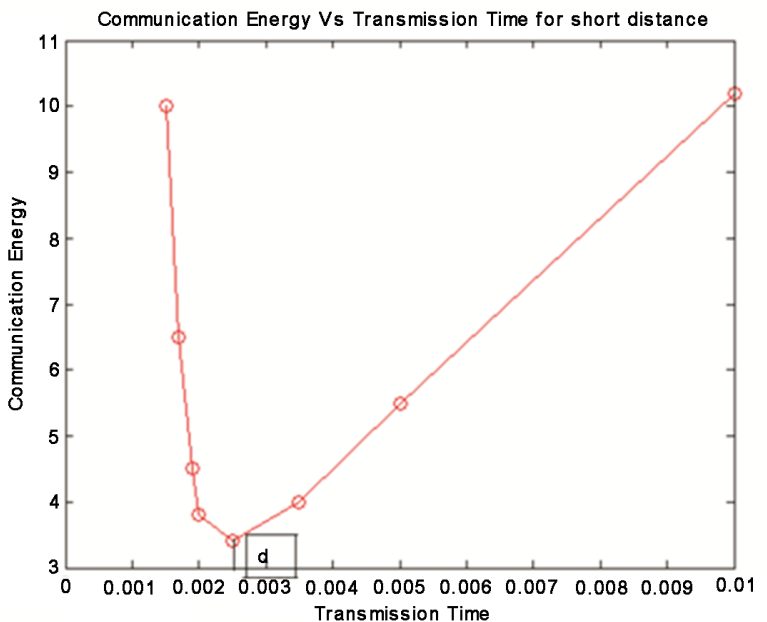

Figure 7. Energy consumption Vs Transmission time (w.r.t distance).

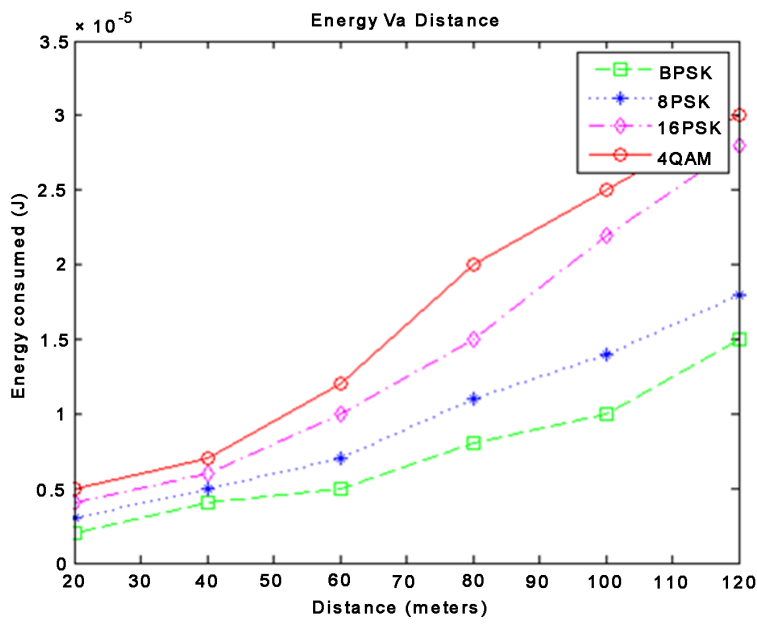

Figure 8. Energy consumed by different modulation schemes (w.r.t distance), when $\theta=30^{\circ}$ during clustering.

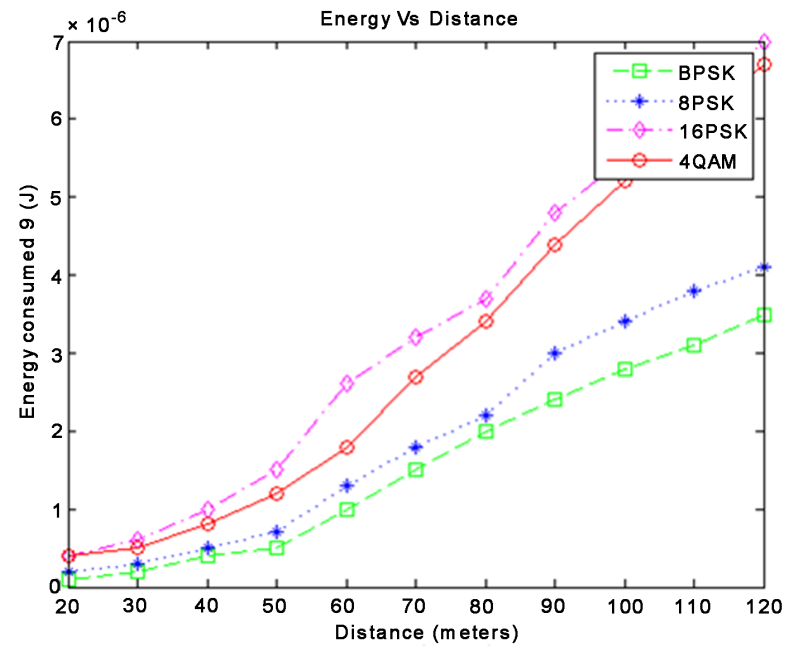

Figure 9. Energy consumed by different modulation schemes (w.r.t distance), when $\theta=45^{\circ}$ during clustering. 
results it is shown that once the uniform clustering at routing layer is done, optimum packet size as well as modulation scheme can be applied on each member of the sensing area to conserve energy of every node of the network. The results presented in this paper may help network designers in a greater way. The main contributions of this paper are as follows:

$\checkmark$ Cross-layer information exchange especially among bottom three layers of OSI model is the best possible way to conserve energy of WSNs.

$\checkmark$ Optimal hop distance (by using uniform clustering) and packet size (without overheads) are two crucial parameters for achieving energy efficiency in WSNs.

$\checkmark$ The entire network operates at the optimum hop distance due to which communication energy becomes independent of channel noise and thereby helps in enhancing Quality of Services (QoS) of the network.

$\checkmark$ To use energy of WSNs in a best possible way, inter layer information exchange among bottom three layers is required. Each node's hop distance in its cluster helps to optimize the data packet as well as its modulation scheme at physical layer.

Future work: This research work can be further extended by exchanging information between transport and application layers. Other than this our future work may include testing of this analysis on hardware (motes) and evaluate the results.

\section{Acknowledgements}

We are thankful to Dr. Baljeet Singh, Associate Professor (Mathematics) for his suggestions and valuable insights.

\section{References}

[1] Srivastava, V. and Motani, M. (2005) Cross-Layer Design: A Survey and the Road Ahead. IEEE Communications Magazine, 43, 112-119. https://doi.org/10.1109/MCOM.2005.1561928

[2] Lin, X.H., Kwok, Y.K. and Wang, H. (2009) Cross-Layer Design for Energy Efficient Communication in Wireless Sensor Networks. Wireless Communication Mobile Computing, 9, 251-268. https://doi.org/10.1002/wcm.608

[3] Akyildiz, I.F., Vuran, M.C. and Akan, O.B. (2006) A Cross-Layer Protocol for Wireless Sensor Networks. IEEE/ ACM Transactions on Networking, 6, 1102-1107. https://doi.org/10.1109/ciss.2006.286630

[4] Luca, C., Riccardo, C. Del, F.G., Luca, M., Vincenzo, M., Luigi, P. and Laura, S.M. (2014) A Cross-Layer Approach to Minimize the Energy Consumption in Wireless Sensor Networks. Lecture Notes in Control and Information Sciences, 331, 309-324.

[5] Zhou, Z.H., Xiang, X.J., Wang, X. and Pan, J.P. (2010) A Holistic Sensor Network Design for Energy Conservation and Efficient Data Dissemination. Computer Networks, 55, 131-146. https://doi.org/10.1016/j.comnet.2010.08.002

[6] Zara, H. and Faisal, B. (2013) XL-WMSN: Cross-Layer Quality of Service Protocol for Wireless Multimedia Sensor Networks. Eurasip Journal on Wireless Communications and Networking, 2013, 174.

http://jwcn.eurasipjournals.com/content/2013/1/174 
https://doi.org/10.1186/1687-1499-2013-174

[7] Fotis, F., Vangelis, G. and Nancy, A. (2008) Cross-Layer Design Proposals for Wireless Mobile Networks: A survey and Taxonomy. IEEE Communications Surveys and Tutorials, 10, No. 1. www.comsoc.org/pubs/surveys

[8] Liu, Q., Zhou, S. and Giannakis, G.B. (2004) Cross-Layer Combining of Adaptive Modulation and Coding with Truncated ARQ over Wireless Links. IEEE Transaction Wireless Communication, 3, 1746-1755.

[9] Cui, S., Goldsmith, A.J. and Bahai, A. (2005) Energy-Constrained Modulation Optimization. IEEE Transaction Wireless Communication, 4, 2349-2360. https://doi.org/10.1109/TWC.2005.853882

[10] Ritesh, M., Cui, S., Lall, S. and Goldsmith, A.J. (2007) Modelling and Optimization of Transmission Schemes in Energy-Constrained Wireless Sensor Networks. IEEE/ ACM Transaction Networks, 15, 1359-1372. https://doi.org/10.1109/TNET.2007.897945

[11] Ye, W., Heidemann, J. and Estrin, D. (2002) An Energy-Efficient MAC Protocol for Wireless Sensor Networks. IEEE Infocom, New York, 23-27 June 2002, 1567-1576. http://www.isi.edu.

[12] Almiani, K., Selvakennedy, S. and Viglas, A. (2008) RMC: An Energy-Aware Cross-Layer Data-Gathering Protocol for Wireless Sensor Networks. 22nd International Conference on Advanced Information Networking and Applications, Okinawa, 25-28 March 2008, 410-417. https://doi.org/10.1109/aina.2008.90

[13] Melodia, T., Vuran, M.C. and Pompili, D. (2006) The State of the Art in Cross-layer Design for Wireless Sensor Networks. Springer Lecture Notes in Computer Science (LNCS), 3883, 78-92. https://doi.org/10.1007/11750673 7

[14] Van Hoesel, L., Nieberg, T., Wu, J. and Havinga, P.J.M. (2004) Prolonging the Lifetime of Wireless Sensor Networks by Cross-Layer Interaction. IEEE Wireless Communications, 11, 78-86. https://doi.org/10.1109/MWC.2004.1368900

[15] Yuan, J., Li, Z., Yu, W. and Li, B. (2005) A Cross-Layer Optimization Framework for Multicast in Multi-Hop Wireless Networks Wireless Internet. First International Conference on Wireless Internet (WICON05), Budapest, 10-15 July 2005, 47-54. https://doi.org/10.1109/WICON.2005.2

[16] Feng, L., Lu, Y., Wei, Z.C., Zhu, Z.X. and Ding, X. (2013) Cross-Layer Power-Control-Based Real Time Routing Protocol for Wireless Sensor Networks. International Journal of Distributed Sensor Networks. Hindawi Publishing Corporation, Cairo.

[17] Ge, W., Zhang, J. and Shen, S. (2007) A Cross-Layer Design Approach to Multicast in Wireless Networks. IEEE Transaction Wireless Communication, 6, 1063-1071. https://doi.org/10.1109/TWC.2007.05468

[18] Choe, H.J., Ghosh, P. and Das, S.K. (2009) Cross-Layer Design for Adaptive Data Reporting in Wireless Sensor Networks. IEEE International Conference on Pervasive Computing and Communications, Galveston, 9-13 March 2009, 1-6. https://doi.org/10.1109/percom.2009.4912844

[19] Korhonen, J. and Wang, Y. (2005) Effect of Packet Size on Loss Rate and Delay in Wireless Links. IEEE Wireless Communications and Networking Conference, New Orleans, 13-17 March 2005, 1608-1613. https://doi.org/10.1109/wcnc.2005.1424754

[20] Hou, Y., Hamamura, M. and Zhang, S. (2005) Performance Trade-Off with Adaptive Frame Length and Modulation in Wireless Network. IEEE International Conference on Computer and Information Technology, Shanghai, 21-23 September 2005, 490-494.

[21] Modiano, E. (1999) An Adaptive Algorithm for Optimizing the Packet Size Used in 
Wireless ARQ Protocols. Wireless Networks, 5, 279-286.

https://doi.org/10.1023/A:1019111430288

[22] Sankarasubramaniam, Y., Akyildiz, I.F. and McLaughlin, S.W. (2003) Energy Efficiency Based Packet Size Optimization in Wireless Sensor Networks. IEEE International Workshop on Sensor Network Protocols and Applications, Anchorage, 11 May 2003, 1-8.

[23] Chen, Y., Sirer, E. and Wicker, S. (2003) On Selection of Optimal Transmission Power for Ad-Hoc Networks. Hawaii International Conference on System Sciences (HICSS-36), Big Island, 6-9 January 2003, 1-10.

[24] Proakis, J. (2001) Digital Communications. McGraw-Hill Book Company, New York, 174-220.

[25] Rappaport, T.S. (2002) Wireless Communications: Principles \& Practice. Pearson Education, London, 294-325.

Submit or recommend next manuscript to SCIRP and we will provide best service for you:

Accepting pre-submission inquiries through Email, Facebook, LinkedIn, Twitter, etc. A wide selection of journals (inclusive of 9 subjects, more than 200 journals)

Providing 24-hour high-quality service

User-friendly online submission system

Fair and swift peer-review system

Efficient typesetting and proofreading procedure

Display of the result of downloads and visits, as well as the number of cited articles

Maximum dissemination of your research work

Submit your manuscript at: http://papersubmission.scirp.org/

Or contactwsn@scirp.org 